\title{
Detection of Sickle Cell Anemia Through Contour Evidence Extraction and Estimation
}

\author{
Aruna N. S., Hariharan S.
}

\begin{abstract}
Diagnosis of sickle cell anemia by manual visual inspection through microscope is time consuming and causes human errors. Observational errors occur mostly due to overlapping of cells in blood smear image. Here, an automatic segmentation approach is introduced which isolates sickle cells from all other cells within a blood sample. The proposed system is an approach to find the elliptically shaped sickle cells through geometric feature extraction and contour based segmentation to isolate sickle cells. This technique is a method of isolating sickle cells from other cells within blood sample using cell morphology. A combined approach of extraction of seed points, contour extraction and estimation of contours is used for separation of sickle cells from red blood cells. The methods used for the extraction of seed points are by Ultimate Erosion for Convex Sets and Fast Radial Symmetry transform. The contour evidence is extracted by associating edges of the cells to the seed points. The overlapping and clustered cells in image are identified using ellipse fitting method for contour estimation. Using the seed points and the contour extraction, the edges of the cells are estimated. The lines joining the shape of cells are drawn through estimation of shape of contour. This eliminates cells other than elliptical shaped cells. The proposed system can successfully isolate sickle cells from healthy blood cells within the blood smear image. This automated system has a better accuracy and faster computation speed compared to the existing methods for the detection of sickle cells. This identification methodology helps the health professionals for faster diagnosis.
\end{abstract}

Keywords :Sickle cell anemia, Seed point, contour extraction, contour estimation.

\section{INTRODUCTION}

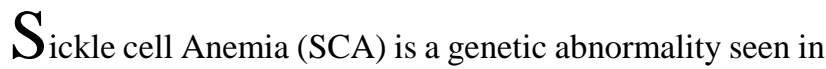
tribal people not only in India but also many countries in the world. This disease is due to a mutation in $\beta$-globin (HBB) gene that informs our body to create the iron abundant compound that creates blood red in color and enables Red blood cells (RBCs) to carry oxygen from lungs throughout our body (hemoglobin). The disease is caused due to deformation within the shape of red blood cells. Sickle cell anemia is

Manuscript received on August 19, 2021.

Revised Manuscript received on August 25, 2021.

Manuscript published on August 30, 2021.

* Correspondence Author

Aruna N S.*, Department of Electrical Engineering, College of Engineering Trivandrum, Kerala, India. Email:arunasurendran2006@gmail.com

Dr. Hariharan S., Department of Electrical Engineering, College of Engineering Trivandrum, Kerala, India Email: harikerala2001@yahoo.com

(c) The Authors. Published by Blue Eyes Intelligence Engineering and Sciences Publication (BEIESP). This is an open access article under the CC BY-NC-ND license (http://creativecommons.org/licenses/by-nc-nd/4.0/) inherited in an autosomal recessive manner. When an individual inherits two abnormal hemoglobin genes which means one from each parent causes sickle cell disease. This is often caused due to the mutation in HBB gene in sickle cells that distorts one among the amino acids, in beta chain of hemoglobin. This defect causes the hemoglobin protein to stick each other and form very hard fibers. These fibers distort the shape of RBCs and make them more fragile. It causes considerable body pain, swelling in hand and feet, stroke and bacterial infections [1,2]. During a study conducted on 2015, around 4.4 million people have sickle cell disease, and an additional 4.3 million have sickle cell trait.

The existing method for diagnosis of anemia might not provide a correct result even after an exhaustive testing. Visual assessment of the discoloured RBCs is additionally taken for diagnosis of anemia which is discussed by Bain[3] and Thirusittapalam[4]. There are various types of deformation in RBCs, which is confusing during the assessment of disease diagnosis. Christoph[5] and Maciaszek[6] discussed the drawbacks occurring in microscopic result and manual inspection which are time consuming and any interruption in detection process leads to erroneous diagnostic results. On visual inspection of image, the lab technician is most likely to get confused with the rare anemic conditions of disease. If the counting process is interrupted, the medical laboratory technologist should start from the scratch. After the blood cell slides have been analysed they are washed away. Thus there is no quick and simple method of retrieving the blood cell images for storage or future reference. All blood samples that come for testing are not saved as images. Cases of rare medical condition are stored as images for future references.

Image processing of blood smear image helps in reducing the observational defects in visual inspection of blood image. In this paper, a method was introduced for fast and accurate detection of sickle cells in blood image. However, there have been researches conducted in image processing to classify sickle cells [7-15] by different shape deformations of RBCs. This paper describes a much faster image processing of blood smear images. All unwanted elements within a blood smear image are removed by suitable algorithm. This also involves removing low frequency noise, normalization of intensity of each particle in the image, removing the reflections and masking selective portions of the images. Image filtering includes linear, nonlinear, edge detection and zooming operations. Brightness and contrast of images can be adjusted by enhancement methods.

Published By:

Blue Eyes Intelligence Engineering 


\section{Detection of Sickle Cell Anemia Through Contour Evidence Extraction and Estimation}

After comparison of filtered images with various filters, median filter [16] fits the most effective filter suitable for processing of SCA. Enhancement of images is performed through process of contrast improvement, image sharpening, suited for further processing or analysis. This is often used for point processing, filtering and image pseudo colouring. After image enhancement and morphological operations are applied, other components of blood such as WBCs and platelets can be easily removed [17]. The edge detection of the sickle cells determines the presence of edges or lines in blood smear images thus outlining them in an appropriate way. The orientation, amplitude and location of particular interested areas are the important characteristics of possible edges. The detector decides edges based on the above mentioned characteristics. Different edge detector operators [18] are also used for the edge detection according to variations in characteristics of images.

Feature extraction of objects within the blood smear image helps to detect relevant and important attributes for further processing. There are multitudes of features that can be chosen for feature extraction. Feature selection involves choosing the best features by excluding irrelevant or redundant features. This also helps in extracting information from data and takes less number of features [10, 19-22]. This removes irrelevant features thus increasing the efficiency, accuracy and enhancing the resulting images. In this paper, a simple feature extraction method is selected based on less processing time. Geometric features itself can distinguish sickle cells from all other objects in the blood smear image. Wang[23] proposed shape from shading method and multi-scale surface fitting to segment RBCs through shape feature extraction. Sklansky[24] presented a set of features that can be implemented for segmentation of continuous tone images using computer techniques. Kanafiah[25] proposed radial based cell formation algorithm for overlapping cell separation. Cell detection based on shape signature using neural networks has been introduced by Elsalamony[26] for distinguishing different shapes of RBCs. The distinctly existing cells in the image can be eliminated through these techniques but overlapping and clustered cells cannot be distinguished. Gonzalez[27] introduced ellipse adjustment and an algorithm to separate the clustered RBCs and sickle cells. Elsalamony[28] proposed cell signature, neural networks and SVM to detect different anemic conditions in human red blood cells and gave an idea about numerical values of features of different shapes of RBCs. A discussion on cell splitting at a high degree of overlapping in blood smear images was done by Ngoc[29].

Park in 2013[30], introduced a segmentation method and classification of overlapping nanoparticles which gave an concept about separating the blood components which are at a nanoparticle dimension. This is an automated morphology analysis of partially overlapping nanoparticles. The methods adopted were particle separation with ultimate erosion process proposed by Loy[31], Cheng[32] and edge-to-marker association for the segmentation of overlapping nanoparticles. Using the identical method Zafari[33] and Kharma[34] attempted to separate elliptical bodies in microscopic image samples. The marking of seed point and edges [35] of every objects and segmentation are discussed by
Kothari[36] through fitting the elliptical shaped bodies. Pilu[37] and Fitzgibbon[38] has discussed about fitting the ellipse using direct least square method. In this paper, the blood sample image consists of round, elliptical, concave and elongated shape objects. A segmentation technique has got to be applied on those objects [39-42] was discussed about methods in identifying concave, ellipse, round and sickle shaped objects for segmentation. Error detection during the estimation of contour during contouring with a completely unique method was discussed to reduce errors during the method $[43,44]$. A comparison of different existing methods was conducted in [45-49] that discusses about segmentation of objects and sickle cells in blood smear image.

\section{METHODOLOGY}

The images that were collected from different laboratories and dataset from different authors were saved to 64 bit Core-i5 Windows supported computer. Those images were converted to different color components like red, green and blue. Qualitatively green color encompasses a darker appearance with good contrast between different components in blood and also the background. Thus green component images were selected for further processing of images. The image processing techniques for the segmentation of sickle cells from other cells within the proposed system are mentioned below:

\section{A. Filtering}

For the visualization and interpretation of every image for good quality medical images, filtering of images is critical. There are a variety of filtering methods available for different applications. Major part in filtering is that the removal of noise without affecting information that is required for further processing and this also helps within the enhancement of images. Median filters [18] are capable of removing noise by preserving edges. Here, each pixel in image was considered successively and it is the very near neighbors to make a decision whether it is the representative of its surroundings. This filter replaces the pixel values with the median of its neighboring pixel values. The kernel is going to be a 3x3 matrix with all elements 0.1111 .

\section{B. Edge Detection}

The shape of an object can be determined by the identifying the edges of each object. Edge detection helps in finding the edges and adding those edges on images to get a darkened with sharp edge. This gives more information about the features of the object and preserves the useful data in the image. Canny Edge Detection technique [20] satisfies most edge preservation conditions by giving better localization, minimal response and good detection of objects. The basic equation for Canny edge detector is

$$
H(g .)=\int_{-W}^{W} G(-x) f(x .) d x
$$

Published By:

Blue Eyes Intelligence Engineering

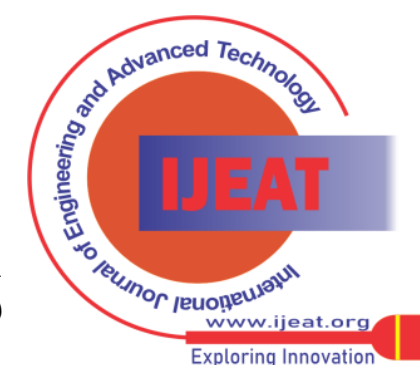


where $f(x)$ is impulse response of noise, $G(x)$ is the edge where edge is centered at $x=0 . H(g$.) represents the response of filter to center of edge and filter bounded at finite impulse response $-W$ and $W$.

\section{Feature Extraction}

Extraction of features minimizes the quantity of resources that are required to expalin an object. The method of feature selection could be a tedious one as some important features which is able to be of high importance but have complicated measurement procedure. The object that has to be extracted from the image was sickle cell and the features can be identified using its shape [21-24]. Selection of the exact feature is a difficult process, so it requires a large study and to seek out the simplest and fast method for extraction of features. Geometric features [12] were suitable for identification of shape of the object.

- Geometric Features: Area, centroid, maximum axis length, minimum-axis length, eccentricity, perimeter, form factor, convex- area were some of the main features of geometric feature. Table1 mentions different variables selected for geometric features of objects.

Table.1 Variables description of geometric features

\begin{tabular}{|l|l|l|}
\hline $\begin{array}{l}\text { Sl. } \\
\text { no. }\end{array}$ & Variables & Description of variables \\
\hline 1 & Area & the actual number of pixels in the region \\
\hline 2 & Major Axis Length & the length of major axis of ellipse in pixels \\
\hline 3 & Minor Axis Length & the length of minor axis of ellipse in pixels \\
\hline 4 & Eccentricity & $\begin{array}{l}\text { the ratio of minor axis length to major axis } \\
\text { length }\end{array}$ \\
\hline 5 & Perimeter & $\begin{array}{l}\text { the distance between each adjoining pair of } \\
\text { pixels around the edge of the region }\end{array}$ \\
\hline 6 & Form factor & $\begin{array}{l}\text { the distance between adjoining pair of pixels } \\
\text { on the border of the region }\end{array}$ \\
\hline 7 & Convex Area & the area of convex hull of a region \\
\hline
\end{tabular}

\section{Extraction of Seed Points}

Seed points are identified to find the number of individual elements in an image. The seed points are extracted using an iterative algorithm Ultimate Erosion for Convex Sets(UECS)[29] and Fast Radial symmetry Transform(FRS) [30].

- Ultimate Erosion for Convex Sets (UECS): Let there be $n$ objects in an binary image $I$. with a set of pixels in interior or boundary of object $i$ be $C_{i}$. given that $I=U_{i .=1}^{n} C_{i}$. A pairwise disjoint markers were applied on the image to obtain a connected subset for $C_{i}$. Those markers were obtained by performing Minkowski subtraction [31] to $I$. for $B .(0,1)$ where $B .(x, r)$ for a closed ball centered at $x$ with radius $r$. By repeated erosion operation separates the overlapping junctions. A complete set of erosion operation was carried out to the remaining connected sets, which results in markers hence called as Ultimate Erosion. This process leads to over segmentation of objects and this can be eliminated through Ultimate Erosion of Convex sets.

Ultimate erosion approach uses iterative process starting from $I$. to update till $I^{(t)}$. The process starts with $I^{0}=I$. For $i^{\text {th }}$ connected component $A_{i}^{(t-1)}$ in $I^{(t-1)}$, to compute $R_{i}$ and updating $I^{(t-1)}=U_{i} R_{i}$.

$$
R_{i .}=\left\{\begin{aligned}
A_{i .}^{(t-1)} \Theta B_{.}(0,1) & \text { if } A_{i .}^{(t-1)} \text { isnotconvex } \\
A_{i .}^{(t-1)} & \text { otherwise }
\end{aligned}\right.
$$

And iteration stops at $I^{t}=I^{(t-1)}$. To find the concavity of object in image suppose that the image $I$. be a connected set, if $O=\operatorname{conv}(I$.) and $\mathrm{V}=\mathrm{O} / \mathrm{I}$. Then consider $V$ has $m$ connected sets. The boundary of $\mathrm{j}^{\text {th }}$ connected set of $V_{j}$, the size of concavity of $V$ is

$c(V)=\max _{j=1 \ldots m}\left(\frac{d\left(V_{j} \cap \partial O, V_{j} \cap \partial I\right.}{l(L)}\right)$

Wher $\partial I=\max _{x} \min _{y}\|x-y\|$ and $l(L)=l\left(V_{j} \cap \partial O\right)$, the length of line segment $L$. The concavity range measurement is $[0,0.5]$. The criteria for stopping iteration was comparing the threshold value[37].

- Fast Radial symmetry Transform (FRS): To find the seed points in blood such as RBCs and sickle was done by Bounded Erosion method followed by Fast Radial Symmetry Transform $[30,33]$ is used in work to improve the accuracy of extracting seed points. FRS transform eliminates the duplicate seed points by evaluating the extracted seed points for rotational symmetry. A continuous set of radii can be calculated through this transform and it will point out every edge pixels of the image space.

In this transform, for each radius $m$. an orientation projection image denoted as $O_{m}$ : and the magnitude projection image denoted as $M_{m}$. were formed. The orientation image and magnitude images are formed taking the gradient $g$ at each point $p .(i, j)$ from which the corresponding positive affected pixel $p_{+v e}(i, j)$ and negative affected pixel $p_{-v e}(i, j)$ are determined. $p_{+v e}(i, j)$ is the pixel that $p(i, j)$ is pointing to the distance $m$. away from $p$ and $p_{\text {-ve }}(i, j)$ is the pixel distance $m$. away with the gradient which is pointing directly away from it. The coordinates are given by

$\operatorname{div}\left(e_{j}, S_{i}\right)=\min _{x \in S_{i}} \frac{\vec{g}\left(e_{j}\right) \cdot \vec{l}\left(x, e_{j}\right)}{\left\|\vec{g}\left(e_{j}\right)\right\|\left\|\vec{l}\left(x, e_{j}\right)\right\|}$

$p_{-v e}(i, j)=(i, j)-$ round $\left(\frac{g(i, j)}{\|g(i, j)\|} \cdot m\right)$

Orientation projections coordinates of images were

$O_{m}\left(p_{+v e}(i, j)\right)=O_{m}\left(p_{+v e}(i, j)+1\right.$

$O_{m}\left(p_{-v e}(i, j)\right)=O_{m}\left(p_{-v e}(i, j)-1\right.$

Magnitude projection coordinates of images were

$M_{m}\left(p_{+v e}(i, j)\right)=M_{m}\left(p_{+v e}(i, j)+\|g(i, j)\|\right.$
$M_{m}\left(p_{-v e}(i, j)\right)=M_{m}\left(p_{-v e}(i, j)-\|g(i, j)\|\right.$

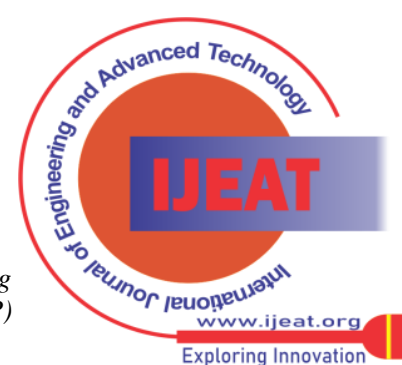




\section{Detection of Sickle Cell Anemia Through Contour Evidence Extraction and Estimation}

A radial symmetry $S_{m}$. was calculated for above obtained images with radius $\mathrm{m}_{\mathrm{g}} \varepsilon\left[\mathrm{R}_{\min }, \mathrm{R}_{\max }\right]$ using convolution of $F_{m}$ and a two dimensional Gaussian $A_{m}$. It is given by

$S_{m .}=F_{m .} * A_{m}$.

Where

$F_{m .}(i, j)=\frac{M_{m .}(i, j)}{k_{m .}}\left(\frac{\left|O_{m .}(i, j)\right|}{k_{m .}}\right)^{\alpha}$

$\alpha$ is radial strictness and $k_{m}$. is scaling factor which normalize $M_{m}$. and $O_{m}$. on different radii.

Hence $S$ was found to be

$S=\frac{1}{\left|N_{m . \in\left[R_{\min }, R_{\max }\right]}\right|} \sum S_{m}$

$N$ is the set of radii which are radially symmetric features that has to be detected. The output image $S$ obtained will have positive values that correspond to regions with bright radial symmetry and negative values indicate the regions with dark symmetry.

\section{- Contour Evidence Extraction: The contour}

extraction is processed by edge to seed point association by taking the parts of overlapping objects and those detected seed points. The contour estimation includes edge to seed point combination method by distance and divergence index to assign edge pixel points to that seed points. When the seed points are marked through seed point extraction method, the next step grows the markers on repeated application of geodesic dilations to those markers [31,33,35]. The growth stops when these markers collide with other grown markers. These markers infer as contour evidence and are the complete contours of the objects. In contour evidence extraction, first step is to extract all the edge pixels from an image and then associate those pixels with each individual marker according to the distance measurement.

The distance measurement was given by

$g\left(e_{j}, S_{i}\right)=\min _{x . \in S_{i}} g_{j}(x)$

Where $g_{\mathrm{j}}(x)$ is Euclidean distance $\left|e_{j}-x\right|$, the line $e_{j}$ to $x$ resides entirely within $I$ and $\infty$ for line outside $I$. This method avoids overemphasizing and irrelevant marking. The divergence index of $e_{j}$ from $S_{i}$, compares the direction of intensity gradient at $e_{j}$ along the direction of line from $x \varepsilon S_{i}$ to $e_{j}$ [33]. This is expressed in a cosine function given by

$\operatorname{div}\left(e_{j}, S_{i}\right)=\min _{x \in S_{i}} \frac{\vec{g}\left(e_{j}\right) \cdot \vec{l}\left(x, e_{j}\right)}{\left\|\vec{g}\left(e_{j}\right)\right\|\left\|\vec{l}\left(x, e_{j}\right)\right\|}$

where $\vec{g}\left(e_{j}\right)$ is the direction of intensity gradient at $e_{j .}$ and $\vec{l}\left(x, e_{j}\right)$ is the line direction from $x \in S_{i}$ to $e_{j .}$. For these images, the intensity of objects is less than the background intensity. So, if $e_{j}$. is a part of $C_{i}$ contour, the gradient at $e_{j}$. diverges from $S_{i}$. $C_{i}$ is a convex object; the gradient direction is very close to vector direction that is from $S_{i}$ to $e_{j}$. is the cosine of angle between those two directions which are close is being maximized. The divergence index is the cosine of angle between two consecutive vectors.

The relevance measure is

$\operatorname{rel}\left(e_{j .}, S_{i}\right)=\frac{1-\lambda}{1+g\left(e_{j .}, S_{i}\right) / n I t e r}+\lambda \frac{\operatorname{div}\left(e_{j .}, S_{i}\right)+1}{2}$

The weight constant $\lambda \varepsilon[0,1]$, nIter is the number of erosion iterations which are normalised to $[0,1]$. For experimental results $\lambda$ was chosen to be 0.5 .

Figure 1(a) shows the original image. Figure 1(b) shows the binary image of the original image after the removal of RBCs, WBCs and platelets. The image consists of sickle cells, overlapped cells and clustered cells. This image also shows the rectangular marking where the image is cropped. Figure 1 (c) is the cropped image. Figure 1(d) shows the marking of the seed point of

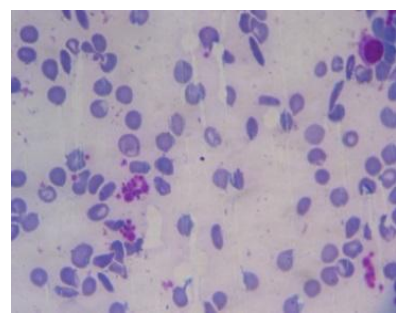

(a)

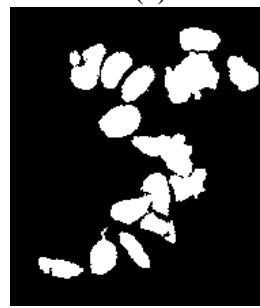

(c)

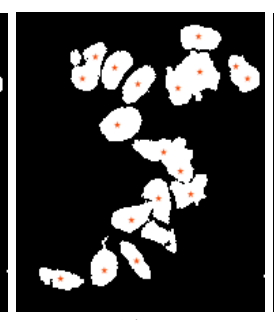

(d)

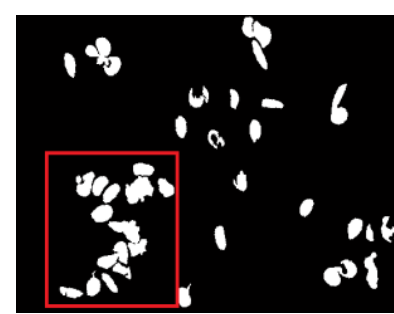

(b)

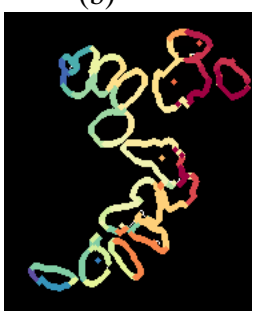

(e)
Fig 1. Extraction of contour evidence with edge to seed point association applied on the cropped portion of image (a) original image (b) cropped area marked on the binary image (c) cropped image (d) plot of seed point on each object (e)contour evidence extraction and estimation using edge to seed point method

each cell. Figure 1(e) shows the extraction of contour evidence and estimation of contour using edge-to-seed point method and contours drawn for edge of each object.

- Contour Estimation: Finding the missing parts in image where contour evidence that estimated was performed by contour estimation. Contour evidence could not separate the overlapping and clustered cells during its operation.

Ellipse fitting was included in contour estimation to segment sickle cells.

A set of data points $(u, v)$, where an objective cost function that is characterizing the goodness of ellipse is optimized based on algebraic deviation. In ellipse fitting method [36-39], the ellipse is formulated by the zero set of a 2nd order polynomial equation. Then for a given point $(u, v)$, the ellipse with a parameter vector a is defined by

$a_{0} u^{2}+a_{1} u v+a_{2} v^{2}+a_{3} u+a_{4} v+a_{5}=0$

where

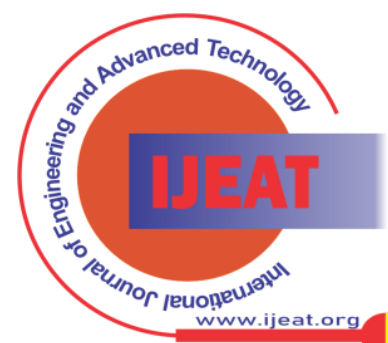


$a=\left[\begin{array}{llllll}a_{0} & a_{1} & a_{2} & a_{3} & a_{4} & a_{5}\end{array}\right]$

The ellipse of quadratic condition is given by

$\Delta=a_{1}^{2}-4 a_{0} a_{2}<0$

In ellipse fitting the sum of squared algebraic distances were involved and was given by

$d(a,(u, v))=a_{0} u^{2}+a_{1} u v+a_{2} v^{2}+a_{3} u+a_{4} v+a_{5}$

And the quadratic condition in matrix was given by

$a^{T} C a<0$

With a constraint matrix

$$
C=\left[\begin{array}{cccccc}
0 & 0 & -2 & 0 & 0 & 0 \\
0 & 1 & 0 & 0 & 0 & 0 \\
-2 & 0 & 0 & 0 & 0 & 0 \\
0 & 0 & 0 & 0 & 0 & 0 \\
0 & 0 & 0 & 0 & 0 & 0 \\
0 & 0 & 0 & 0 & 0 & 0
\end{array}\right]
$$

This minimizes the problem of adopting generic polynomial equations. A specific auxiliary condition has to be considered to get the final solution. The quadratic condition that replaces the direct least square ellipse fitting is given by

$\Delta=a_{1}^{2}-4 a_{0} a_{2}=-1$

Then the system of equations become

$S a=\lambda C a$

$a^{T} \mathrm{Ca}=-1$

This can be obtained by a rank deficient generalized eigen system using the parameter vector $a$. The formulation of ellipse fitting makes computationally efficient and was robust to noise.

\section{RESULTS AND DISCUSSION}

The proposed sickle cell diagnostic system uses different stages of image processing techniques to get an accurate disease identification methodology. The objective of this work is to eliminate normal cells within image and isolate sickle cells. The acquired images in the computer were samples of blood smear consisting of all components of blood such as WBCs, RBCs, platelets and other components. These cells are clustered and overlapped with each other. The isolation of sickle cells using segmentation process is difficult on clustered and overlapped cells as they contain normal cells attached to sickle cells. The sickle cells are isolated to measure disease acuity. Therefore the images have to undergo different pre-processing techniques namely image filtering, enhancement, edge detection, feature extraction, extraction of seed points, extraction of contour evidence and contour estimation which is proposed in this disease identification methodology.

The images in dataset were captured with a Canon Power Shot G5 camera USB connected coupled to an optical laboratory microscope. The blood sample which is also called blood smear is the specimen observed under the microscope. This image is digitized from optical image with 40 times objective lenses which is around 400 magnifications for studying cells and cell structure. These captured images were stored in the computer and an USB connected to the camera.

All images are in JPEG format with 24 bit color depth, resolution 2592 x 1944 . The images were then converted to PNG format to get a more data clarity which is good for pre-processing. The ground truth images marking sickle cells were provided by Histopathology department in Government Medical College Hospital, Kozhikode, Kerala, India and sample images from pathology lab at Government Medical College Hospital, Thiruvananthapuram, Kerala, India. Another set of data were provided by Gonzalez, Guerrero-Pena, S. Herold-Garcia et.al.[27]. The blood smear images acquired from microscope were filtered to remove unwanted elements and noise in the images. Filtering of image uses median filtering as it gives a smoothed image without losing the vital information. The filtered images were used for edge detection of cells by edge detection operators. This information gives the boundary line of each cell to identify the clustering, overlapping and isolated cells in the image. To analyze or identify cells in the image, it requires an accurate foreground cell extraction method making the image segmentation reliable. Various morphological operations such as erosion and dilation were initiated to remove large size and tiny cells from image foreground. All large size cells within the image are WBCs and tiny cells are platelets. The size of WBC and platelets are compared with size of RBCs. Thus removal of WBCs and platelets were initiated.

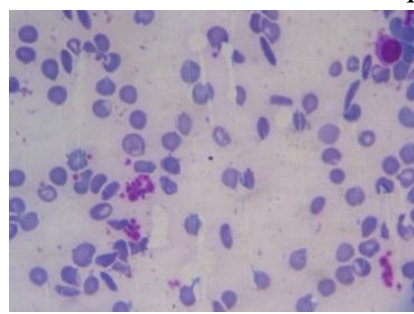

(a)

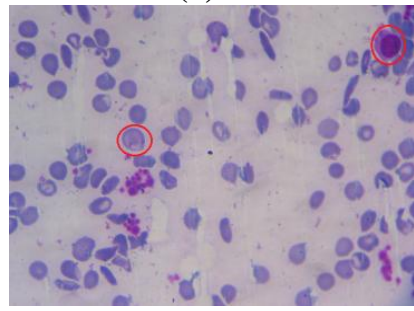

(c)

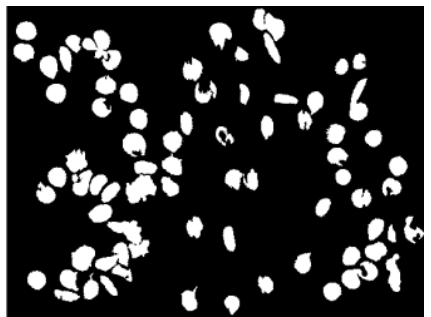

(e)

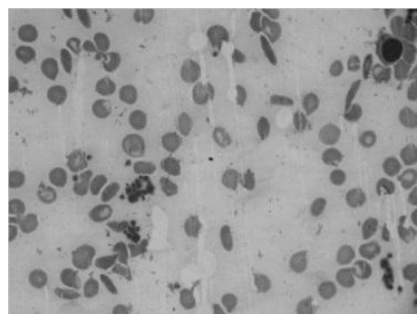

(b)

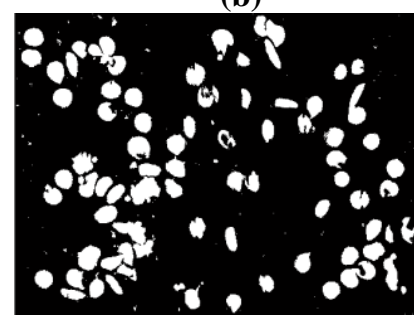

(d)

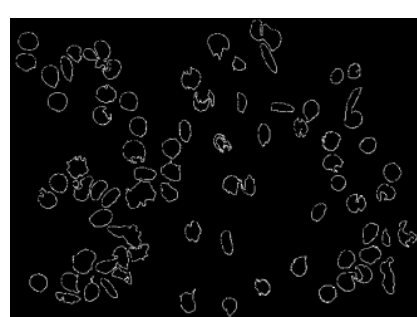

(f)

Fig 2. (a) Original image (b) Green Component image (c) WBCs marked on original image (d) WBCs removed (e) Platelets removed and objects boundary eliminated (f) Edge Detection using Canny detector

Published By:

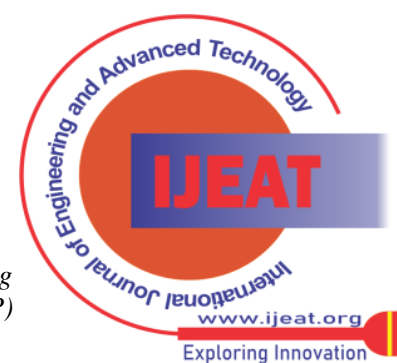




\section{Detection of Sickle Cell Anemia Through Contour Evidence Extraction and Estimation}

White blood cells are identified by their size and shape. These cells are identified by their large size than RBCs and platelets. Figure 2(a) shows the original image. Figure 2(b) is the green component image of original image and figure 2(c) is the WBCs marked for reference.

The green component image has higher contrast which distinguishes WBCs, RBCs and platelets.

The green component image is converted to gray-scale image and then converted to binary image. Binary conversion is performed by Otsu [19] process that finds the threshold value of an image that minimizes the weighted sum within class of variance of each cell. The image erosion process removes irrelevant sized cells from binary image. It is used for shrinking, thinning, stripping away extrusions and separating the joined cells in image. Large cells in the images were eliminated during erosion process. Figure 2(d) shows the image in which WBCs were removed during erosion process. Very small particles within the images are referred as platelets in comparison with the size of RBCs. Platelets are removed by the process of image dilation. The dilation process grows and thickens the cells that are small in size. Through morphological operations using MATLAB functions, platelets are removed. The cells on the boundary of the images were also removed because they will give erroneous information about cells while performing image segmentation. Figure 2(e) shows the image where platelets and cells on the boundary of images are removed.

The image obtained consists of RBCs and sickle cells. For further processing of images, it requires more details of cells present in it. An edge detection technique is applied to get clear edges so that sickle cells can be isolated easily within the image. Canny edge detection operator is utilized for this purpose. The Canny edge detector first blurs the edge and then smoothen it. The edge lines are thick and clearly show the overlapped and clustered cells within the image. Figure 2 (f) shows the edge detection applied to the image using Canny edge detector.

There are around 42 features [12] that can be extracted from blood cell images for identification of blood cells. These include10 metric features, optical density features, optical density histogram features, optical density difference histogram features, run length features and co-occurrence features of the image. In this present work, the extraction of geometric features of an image in proposed methodology makes disease identification easier and faster. The main geometric features are area, centroid, major axis length, minor axis length, eccentricity, perimeter, form factor, convex area. Different geometric features were extracted and compare to find the best feature that can be selected to identify sickle cells from normal blood cells.

The minimum value of RBC eccentricity and form factor is approximately zero, which makes the cells round in shape. Table 2 shows the range of variables selected within feature extracted image, from which the values of eccentricity of RBC is found to be 0.13 to 0.8 and 0.9 to 0.99 for sickle cells. Thus with this particular range of values, it is possible to eliminate RBCs from the blood smear image. The images also contain sickle cells with overlapped and clustered cells. The overlapped and clustered cells also contain RBCs which are overlapped with sickle cells. This overlap needs to be segmented using an easy and fast segmentation method.

Table 2. Range of variables in feature extraction

\begin{tabular}{|c|c|c|c|c|c|c|c|c|c|c|c|c|c|c|}
\hline \multirow[t]{2}{*}{ Object } & \multicolumn{2}{|c|}{$\begin{array}{l}\text { Area in pixel } \\
\text { values }\end{array}$} & \multicolumn{2}{|c|}{$\begin{array}{l}\text { Major } \\
\text { Length }\end{array}$} & \multicolumn{2}{|c|}{$\begin{array}{l}\text { Minor Axis } \\
\text { Length }\end{array}$} & \multicolumn{2}{|c|}{ Eccentricity } & \multicolumn{2}{|c|}{$\begin{array}{l}\text { Perimeter in } \\
\text { pixel values }\end{array}$} & \multicolumn{2}{|c|}{ Form factor } & \multicolumn{2}{|c|}{ Convex Area } \\
\hline & Max & Min & Max & Min & Max & Min & Max & Min & Max & Min & Max & Min & Max & Min \\
\hline RBCs & 76375 & $\begin{array}{l}1159 \\
0\end{array}$ & 150 & 131.25 & 150 & 135 & 0.8 & 0.13 & 1094 & 666.25 & 0.39 & 0.17 & 31107 & 12778 \\
\hline $\begin{array}{l}\text { Sickle } \\
\text { Cells }\end{array}$ & 8469 & 338 & 240 & 190.36 & 100 & 65.88 & 0.99 & 0.90 & 735 & 680 & 0.5 & 0.4 & 20279 & 11098 \\
\hline
\end{tabular}

The isolated erythrocyte cells (RBCs) were eliminated using dilation process by taking the feature extraction values. The resulting output images still have sickle cells, clustered cells and the overlapped cells. An improved faster method was introduced for the segmentation of sickle cells from clustered and overlapped blood cells. The first step for this process involves extraction of seed points in order to differentiate the cells within the images. This result was achieved by Bounded Erosion Fast radial Symmetry transformation and Ultimate Erosion for convex sets method [29-32]. The cells within the images were differentiated by their size and shape like elliptical/elongated and round objects. The clustered and overlapped cells were measured to find the seed points of the objects and then the seed points were marked at the center of each cell. Then the seed points of

objects and the seed points were marked on the center of each object.

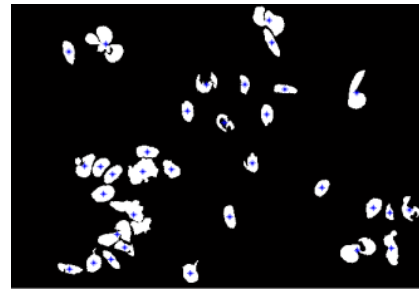

(a)

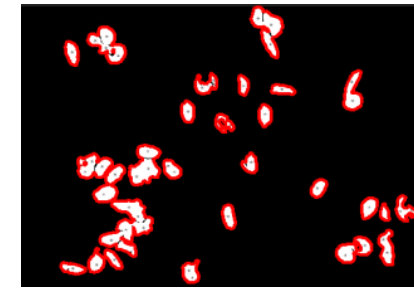

(b)

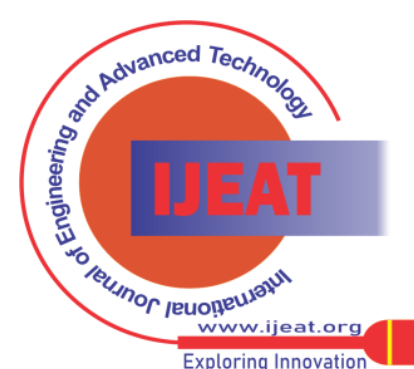




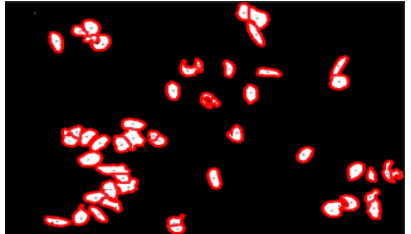

(c)

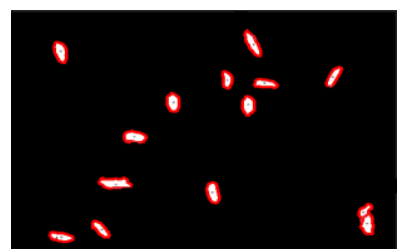

(d)
Fig 3. (a) Seed point extraction (b) Contour evidence extraction (c) Contour estimation (d) Isolated Sickle cells

Figure 3 (a) shows the seed points marked on the objects in the image. The seed points obtained on each cell should be positioned at the center of cell envelope. This was accomplished by using contour evidence extraction and detection of the edge of the object. The associations of seed point to edge pixels are carried to determine the shape of object. Boundary markers were used to discriminate geodesic dilations to the markers. The markers grow according to the edge pixels of the cells in image and the growth stops when the markers collide with other growing markers. These markers were termed as contour evidence. Figure 3(b) shows the image after contour evidence extraction.

The contour estimation of images was performed to find the missing cell contour within the image, where contour evidence was estimated. The overlapped cells were not separated during the contour evidence extraction method. An ellipse fitting method is employed in which the partially observed cells were modeled forming elliptical shape. The direct least square method for ellipse fitting was adopted for estimation of cells within the image. After the extraction of contour evidence, the ellipse fitting method will completely mark the shapes of the cells and hence be able to separately detect each cell within overlapped and clustered cells. This was observed figure 3(c) shows the image that consists of round and elliptical shaped objects. Hence we are able to visually separate sickle cells within the image. Figure 3(c) shows we have isolated sickle cells by eliminating all other normal cells within that image. Figure 3(d) shows the image of isolated sickle cells. Thus it was able to isolate sickle cells from other normal cells within the image.

Thus the proposed diagnostic methodology isolated sickle cells from other normal cells within the image, hence providing health professionals a better idea about the severity of illness. When number of sickle cells is more, then the illness is severe and when it is less, it concludes that it is the starting stage of illness. This also helps professional for appropriate interpretation and fast decision making during treatment procedures.

A study conducted takes into account an isolated RBC and sickle cell from the image shown in figure 4(a) for further clarification in selection of feature variables. The selected cells are shown in image with red rectangular markings. Figure 4(b) shows the image of a cropped RBC and figure 4(c) shows the cropped image of an isolated sickle cell. Figure 4(d) shows the Major axis length and Minor axis length marking on edge detected image of cropped RBC and figure 4(e) shows the Major axis length and Minor axis length marking on cropped sickle cell image.

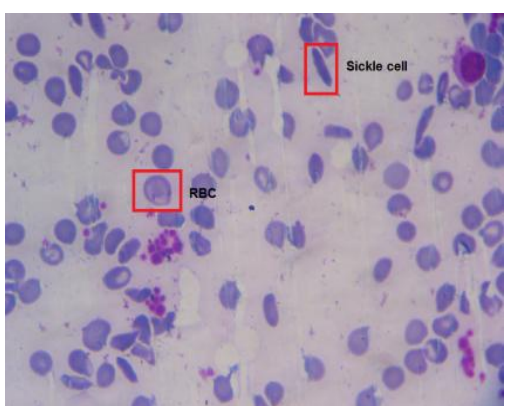

(a)

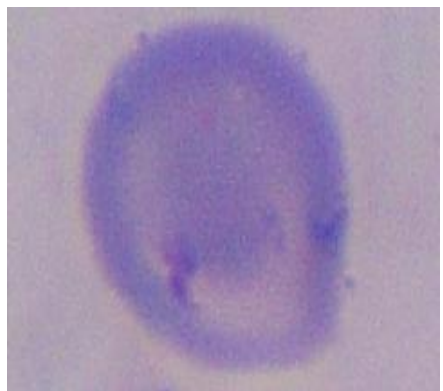

(b)

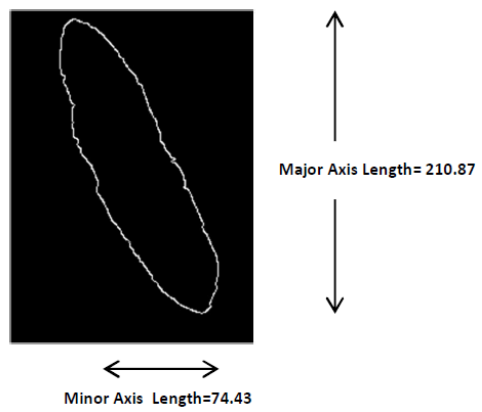

Minor Axis Length=74.43

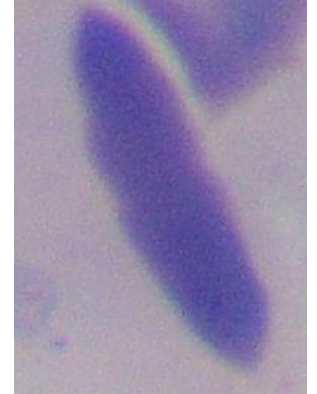

(c)

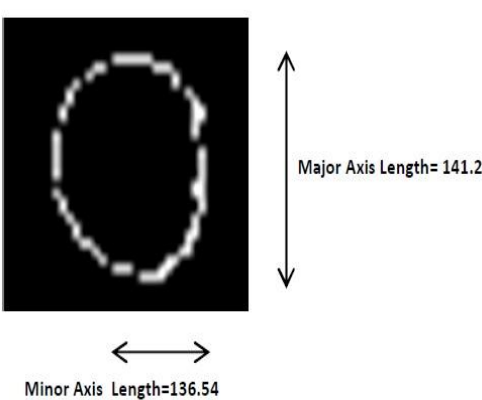

(d)

Fig 4. (a) Selected sickle in original image (b) cropped image of RBC (c) cropped image of sickle (d) Marking Major and Minor Axis on contour image of selected RBC (e) Marking Major and Minor Axis on contour image of selected Sickle cell

The features of cropped RBC and sickle cell were extracted and values that were found are shown in table 3 . Value of eccentricity can easily differentiate sickle cells from RBCs. Table 2 shows the ranges of features of all cells and selected minimum value and maximum values of the features. Table 3 shows the range of feature values of single cells for further clarification. If the observed eccentricity value of an ellipse is close to 1 (like 0.8 or 0.9 ) the ellipse is long and skinny, it is classified as sickle cell. If the observed eccentricity is close to 0 or 0.6 , ellipse is circular and is a normal RBC.

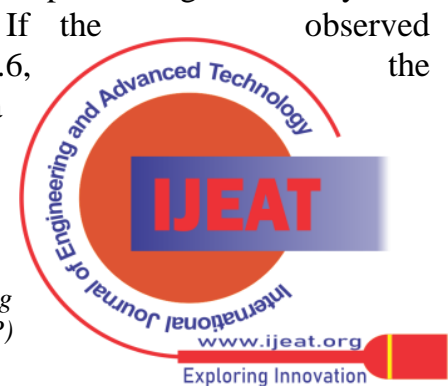




\section{Detection of Sickle Cell Anemia Through Contour Evidence Extraction and Estimation}

If the observation shows area of an ellipse is 20000 and 20000 and above the ellipse is round, it is classified as RBC.

below, it is classified as sickle cell. If observed value of area is

Table 3. Feature values of single RBC and sickle cell

\begin{tabular}{|c|c|c|c|c|c|c|}
\hline Object & $\begin{array}{c}\text { Area in } \\
\text { pixel values }\end{array}$ & $\begin{array}{c}\text { Major Axis } \\
\text { Length }\end{array}$ & $\begin{array}{c}\text { Minor Axis } \\
\text { Length }\end{array}$ & Eccentricity & $\begin{array}{c}\text { Perimeter in } \\
\text { pixel values }\end{array}$ & $\begin{array}{c}\text { Form } \\
\text { factor } \\
\text { Area }\end{array}$ \\
\hline RBC & 36677 & 141.22 & 136.54 & 0.61 & 431.39 \\
\hline Sickle cell & 2910 & 210.87 & 74.43 & 0.93 & 705.21 \\
\hline
\end{tabular}

\section{QUANTITATIVE AND COMPARATIVE STUDY}

Assessment of the experimental results was conducted to understand quantitatively the measure of accuracy of the proposed system. The measurement was done with 300 images from the dataset, 10 images were taken for performance analysis and four images were compared with other existing methods [45-48] shown in figure 5. The performance measure was conducted using Precision, Recall, F-measure and finding the Accuracy of the system.

TP-True Positive: Sick people are identified sick correctly that is it is correctly identified.
TN- True Negative: Healthy people are identified healthy correctly that is correctly rejected.

FP- False Positive: Healthy people are identified as sick that is incorrectly identified.

FN- False Negative: Sick people are identified as healthy that is incorrectly rejected.

$$
\begin{aligned}
& \text { precision }=\frac{T P}{T P+F P} ; \operatorname{Re} \text { call }=\frac{T P}{T P+F N} ; \\
& F-\text { measures }=2\left\{\frac{\text { precision } \times \text { recall }}{\text { precision }+ \text { recall }}\right\} ;
\end{aligned}
$$

Accuracy $=\frac{T P+T N}{T P+T N+F P+F N}$

\begin{tabular}{|c|c|c|c|c|c|c|c|c|}
\hline $\begin{array}{l}\text { Sample } \\
\text { Image }\end{array}$ & $\begin{array}{l}\text { No. of cells } \\
\text { in image }\end{array}$ & $\begin{array}{c}\text { Correctly } \\
\text { segmented }\end{array}$ & $\begin{array}{c}\text { Under } \\
\text { segmented }\end{array}$ & $\begin{array}{c}\text { Over } \\
\text { segmented }\end{array}$ & Precision & Recall & F-measures & Accuracy \\
\hline 1 & 33 & 33 & 0 & 0 & 1 & 1 & 1 & 1 \\
\hline 2 & 59 & 55 & 2 & 2 & 1 & 0.98 & 0.99 & 0.97 \\
\hline 4 & 151 & 143 & 6 & 2 & 0.99 & 0.96 & 0.97 & 0.99 \\
\hline 5 & 502 & 491 & 38 & 3 & 0.99 & 0.94 & 0.96 & 1 \\
\hline 6 & 97 & 96 & 1 & 0 & 1 & 0.98 & 0.99 & 1 \\
\hline 8 & 244 & 232 & 8 & 4 & 0.98 & 0.97 & 0.97 & 0.98 \\
\hline 9 & 578 & 532 & 33 & 3 & 0.99 & 0.94 & 0.96 & 0.98 \\
\hline 10 & 66 & 63 & 3 & 0 & 1 & 0.95 & 0.97 & 1 \\
\hline & & & & & & Total & 0.9757 & 0.9930 \\
\hline
\end{tabular}

Table4. Performance of Sample images

The performance evaluation was conducted on the data set available and a sample of 10 images is shown in Table 4. As the size of RBCs and sickle cells varies from a range of maximum value to minimum value demonstrated in Table 2. The proposed methodology will have to vary accordingly to identify the cells based on their area (in pixels) for segmentation. Hence there will be cases of over segmentation, under segmentation and correct segmentation during performance evaluation of sample images.
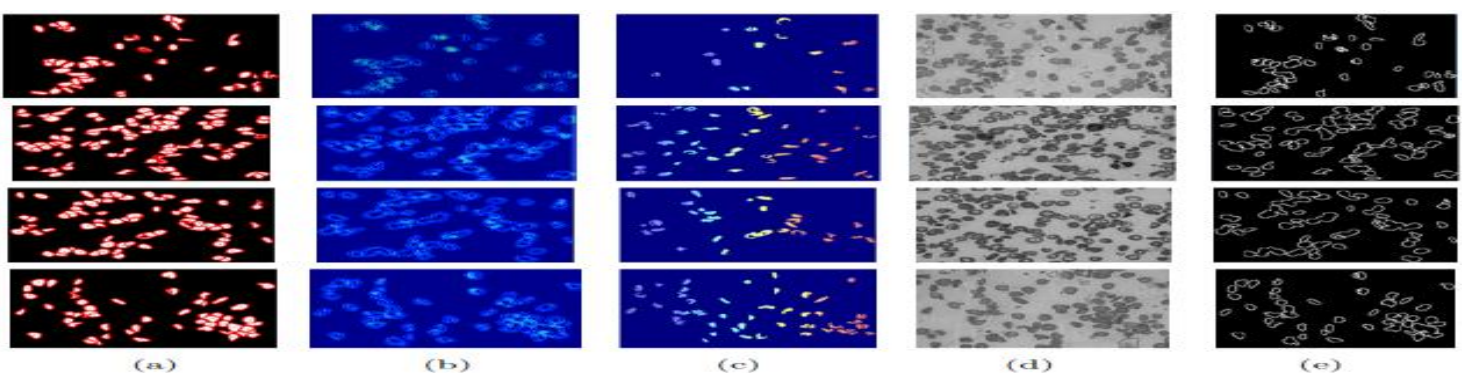

Fig 5. Comparison of proposed system with other four methods (a) Proposed method (b) M1 (c) M2 (d) M3 (e) M4

For comparative study four existing methods were considered and they are M1 as Circular Hough transform, M2 as Watershed Segmentation, M3 as Concave point extraction and M4 as Elliptical curve fitting method. These results in figure 5 shows the proposed system yields superior results
Over-segmentation usually occurs when area of RBC has maximum value and area of sickle has minimum value. Under segmentation occurs when the area of RBC has minimum value and that of sickle cell has maximum value. Correctly segmentation takes place when the touching points of RBC and sickle cell are minimum value and are easily segmented with average values of their area. The accuracy of proposed segmentation was found to be $99.30 \%$. with other methods. Figure 6 demonstrates the plot of computation time of different methods.

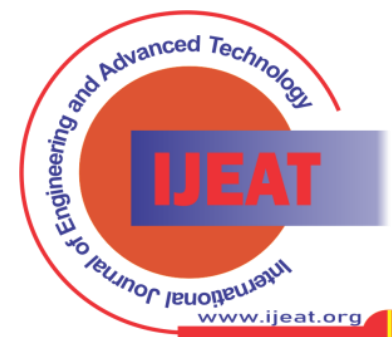


The plot shows that proposed method presented in this paper has much less computation time compared to other methods. The computation time for total processing of the Computer Aided Detection (CAD) system was approximately 10 seconds which is good compared to other existing methods. The proposed work has been carried out in an OS Windows 10 with 64 bit having a built-in software of mathworks RMatlab2014b using intel(R)Core(TM) i5-5005U CPU@2.00GHz processor 4.00GB RAM.

\section{CONCLUSIONS}

A framework for segmentation of sickle cells from blood smear with overlapped and clustered cells is presented. The proposed system consists of different blocks that bring a resulting image with higher accuracy and fast processing. The input original color image was converted to green color component with good contrast between RBCs and background. This image was filtered with median filter as it can preserve the edges and remove noises. This filtered image was converted to gray-scale image and

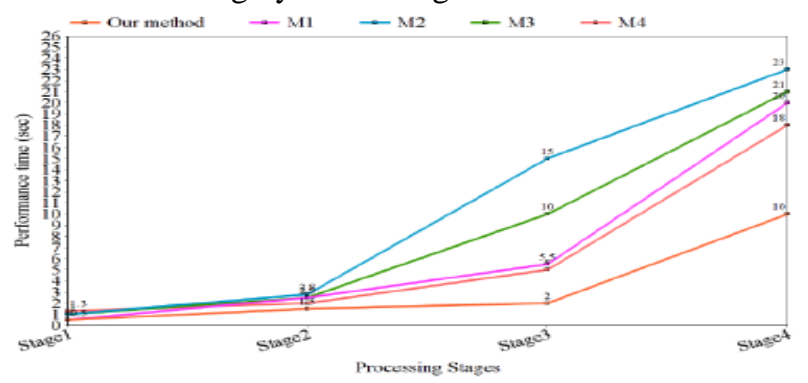

Fig 6. Plot of computation time

then to binary image using Otsu thresholding. The morphological operations were carried out to remove WBCs and platelets. The cells on the boundary of images were removed to avoid erroneous information. The filtered image has been examined with Canny edge detection technique to find edges of the object. Canny edge detector gives a good result and clearly sketch out where the cells are touching, overlapping and isolated.

Isolated cells were separated and removed from the image easily but it was difficult to separate overlapped and clustered cells. Geometric features were extracted and among them eccentricity was chosen to differentiate red blood cells and sickle cells. All the isolated cells of RBCs were removed through segmentation with feature extraction. RBCs and sickle cells that overlap were not identified through that segmentation process. The segmentation by finding the seed point of each cell, extraction of contour edge points and estimation of contour of cells in image makes segmentation easier and faster. The disease identification methodology proposed in this paper, it is possible to isolate sickle cells from RBCs that were overlapped and clustered. The proposed method is compared with existing methods and obtained a higher accuracy and less computation time.

\section{ACKNOWLEDGMENT}

This project was supported by Centre for Engineering Research and Development (CERD) under College of Engineering Trivandrum, Kerala, India.

\section{CONFLICT OF INTEREST}

There is no conflict of interest with respect to material contained in this manuscript.

\section{REFERENCES}

1. Sherman I.J. "The sickling phenomenon, with special reference to the differentiation of sickle cell anemia from the sickle cell trait. Bull. Johns Hopkins Hosp., Nov; 67 (309): 19, 1940.

2. L. Pauling, H. A. Itano, S. J. Singer, and I. C. Wells, "Sickle cell anemia, a molecular disease," Science, vol. 110, no. 2865, pp. 543-548, 1949.

3. B. J. Bain, "Diagnosis from the blood smear," New England Journal of Medicine, vol. 353, no. 5, pp. 498-507, 2005.

4. K. Thirusittapalam, M.J. Hossain, O. Ghita, P. F. Whelan, "A novel framework for cellular tracking and mitosis detection in dense phase contrast microscopy images", IEEE journal of Biomed. Health information vol. 17, pp 642-653 (2013).

5. G. W. Christoph, J. Hofrichter, and W. A. Eaton, "Understanding the shape of sickled red cells," Biophysical Journal, vol. 88, no. 2, pp. 1371-1376, 2005.

6. J. L. Maciaszek, B. Andemariam, and G. Lykotrafitis, "Micro-elasticity of red blood cells in sickle cell disease," The Journal of Strain Analysis for Engineering Design, vol. 46, no. 5, pp. 368-379, 2011.

7. S. A. Bentley and S. M. Lewis, "The morphological classification of red cells using an image analysing computer," British Journal of Haematology, vol. 32, no. 2, pp. 205-214, 1976.

8. Y. Chan, C. Wang, Y. Mao, W. Chang, and K. Lin, "An aneima abnormal red blood cells recognition system,", 2nd int. conf. Chem. Biol. Med. Sci., Phnom Penh, Cambodia, pp. 1-8, 2013.

9. T. S. Chy and M. A. Rahaman, "Automatic sickle cell anemia detection using image processing technique," in 2018 International Conference on Advancement in Electrical and Electronic Engineering (ICAEEE). IEEE, pp. 1-4, 2018.

10. P. K. Das, S. Meher, R. Panda and A. Abraham, "A Review of Automated Methods for the Detection of Sickle Cell Disease," IEEE Reviews in Biomedical Engineering, vol. 13, pp. 309-324, 2020.

11. C. Patgiri and A. Ganguly, "Red Blood Cell and Sickle Cell Detection from Microscopic Blood Images of Sickle Cell Anemic Patient," 2019 International Conference on Wireless Communications Signal Processing and Networking (WiSPNET), pp. 474-478, 2019.

12. L. L. Wheeless, R. D. Robinson, O. P. Lapets, C. Cox, A. Rubio, M. Weintraub, and L. J. Benjamin, Classification of red blood cells as normal, sickle, or other abnormal, using a single image analysis feature," Cytometry, vol. 17, no. 2, pp. 159-166, 1994.

13. H. Lee and Y.-P. P. Chen, "Cell morphology based classification for red cells in blood smear images," Pattern Recognition Letters, vol. 49, pp. 155-161, 2014.

14. C. D. Ruberto, A. Dempster, S. Khan, and B. Jarra, "Analysis of infected blood cell images using morphological operators," Image and Vision Computing, vol. 20, no. 2, pp. 133-146, 2002.

15. M. J. Stuart and R. L. Nagel, "Sickle-cell disease," The Lancet, vol. 364, no. 9442, pp. 1343-1360, 2004.

16. R. Tomari, W. N. W. Zakaria, M. M. A. Jamil, F. M. Nor, and N. F. N. Fuad, "Computer aided system for red blood cell classification in blood smear image," Procedia Computer Science, vol. 42, pp. 206-213, 2014

17. P. Rakshit and K. Bhowmik, "Detection of abnormal findings in human RBC in diagnosing sickle cell anaemia using image processing," Procedia Technology, vol. 10, pp. 28-36, 2013.

18. K. Verma, B. K. Singh, and A. Thoke, "An enhancement in adaptive median filter for edge preservation," Procedia Computer Science, vol. 48, pp. 29-36, 2015.

19. N. Otsu, "A threshold selection method from gray-level histograms," IEEE Transactions on Systems, Man, and Cybernetics, vol. 9, no. 1, pp. 62-66, 1979.

20. J. Canny, "A computational approach to edge detection," in Readings in computer vision. Elsevier, pp. 184-203, 1987.

21. M. Veluchamy, K. Perumal, and T. Ponuchamy, "Feature extraction and classification of blood cells using artificial neural network," American journal of applied sciences, vol. 9, no. 5, p. 615, 2012.

22. M. Nixon and A. S. Aguado, "Feature extraction and image processing for computer vision". Academic Press, 2012

Published By:

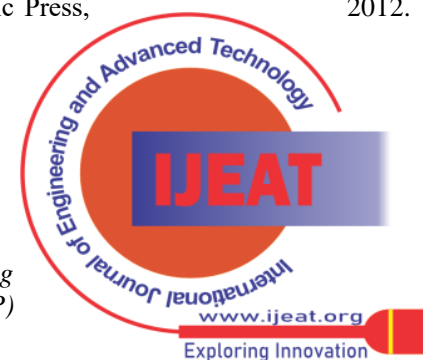




\section{Detection of Sickle Cell Anemia Through Contour Evidence Extraction and Estimation}

23. R. Wang and B. Fang, "A combined approach on RBC image segmentation through shape feature extraction," Mathematical Problems in Engineering, vol. 2012, pp. 1-20, 2012.

24. J. Sklansky, "Image segmentation and feature extraction," IEEE Transactions on Systems, Man, and Cybernetics, vol. 8, no. 4, pp. 237-247, 1978

25. S. N. A. M. Kanafiah, Y. Jusman, N. A. M. Isa, and Z. Mohamed, "Radial-based cell formation algorithm for separation of overlapping cells in medical microscopic images," Procedia Computer Science, vol. 59, pp. 123-132, 2015.

26. H. A. Elsalamony, "Anaemia cells detection based on shape signature using neural networks," Measurement, vol. 104, pp. 50-59, 2017.

27. M. Gonzalez-Hidalgo, F. A. Guerrero-Pena, S. Herold-Garcia, A. J. Capo, and P. D. Marrero-Fernandez, "Red blood cell cluster separation from digital images for use in sickle cell disease," IEEE Journal of Biomedical and Health Informatics, vol. 19, no. 4, pp. 1514-1525, jul 2015.

28. H. A. Elsalamony, "Detection of anaemia disease in human red blood cells using cell signature, neural networks and svm," Multimedia Tools and Applications, vol. 77, no. 12, pp. 15 047-15 074, 2018.

29. Ngoc-Tung Nguyen, Anh-Duc Duong, and Hai-Quan Vu, "Cell splitting with high degree of overlapping in peripheral blood smear," International Journal of Computer Theory and Engineering, pp. 473-478, 2011.

30. C. Park, J. Z. Huang, J. X. Ji, and Y. Ding, "Segmentation, inference and classification of partially overlapping nanoparticles," IEEE transactions on pattern analysis and machine intelligence, vol. 35, no. 3, pp. 1-13, 2013.

31. G. Loy and A. Zelinsky, "Fast radial symmetry for detecting points of interest," IEEE Transactions on Pattern Analysis \& Machine Intelligence, no. 8, pp. 959-973, 2003.

32. J. Cheng, J. C. Rajapakse, "Segmentation of clustered nuclei with shape markers and marking function," IEEE Transactions on Biomedical Engineering, vol. 56, no. 3, pp. 741-748, 2009.

33. S. Zafari, T. Eerola, J. Sampo, H. Kalviainen, and H. Haario, "Segmentation of overlapping elliptical objects in silhouette images," IEEE Transactions on Image Processing, vol. 24, no. 12, pp. 5942-5952, 2015.

34. N. Kharma, H. Moghnieh, J. Yao, Y. Guo, A. Abu-Baker, J. Laganiere, G. Rouleau, and M. Cheriet, "Automatic segmentation of cells from microscopic imagery using ellipse detection," IET Image Processing, vol. 1, no. 1, p. 39, 2007.

35. A. El Allaoui, M. Nasri, "Medical image segmentation by marker-controlled watershed and mathematical morphology," The International Journal of Multimedia \& Its Applications, vol. 4, no. 3, p. 1, 2012.

36. S. Kothari, Q. Chaudry, and M. D. Wang, "Automated cell counting and cluster segmentation using concavity detection and ellipse fitting techniques," in 2009 IEEE International Symposium on Biomedical Imaging: From Nano to Macro. IEEE, pp. 795-798, 2009.

37. M. Pilu, A. W. Fitzgibbon, and R. B. Fisher, "Ellipse-specific direct least-square fitting," in Proceedings of 3rd IEEE International Conference on Image Processing, vol. 3. IEEE, pp. 599-602, 1996.

38. A. Fitzgibbon, M. Pilu, and R. B. Fisher, "Direct least square fitting of ellipses," IEEE Transactions on pattern analysis and machine intelligence, vol. 21, no. 5, pp. 476-480, 1999.

39. X. Bai, C. Sun, and F. Zhou, "Splitting touching cells based on concave points and ellipse fitting," Pattern recognition, vol. 42, no. 11, pp. 2434-2446, 2009.

40. W.-H. Zhang, X. Jiang, and Y.-M. Liu, "A method for recognizing overlapping elliptical bubbles in bubble image," Pattern Recognition Letters, vol. 33, no. 12, pp. 1543-1548, 2012.

41. K. Bikshalu and R. Srikanth, "Segmentation using active contour model and level set method applied to medical images," Int. Journal of Adv. Eng. Global Technology, vol. 4, no. 2, 2016.

42. N. Chaudhary, S. A. Savari, V. Brackmann and M. Friedrich, "SEM Image Denoising and Contour Image Estimation using Deep Learning," IEEE 31st Annual SEMI Advanced Semiconductor Manufacturing Conference (ASMC), pp. 1-6, 2020.

43. X. Yang, R. Seethaler, C. Zhan, D. Lu and W. Zhao, "A Novel Contouring Error Estimation Method for Contouring Control," in IEEE/ASME Transactions on Mechatronics, vol. 24, no. 4, pp. 1902-1907, Aug. 2019.

44. S. Chen, M. Zhao, G. Wu, C. Yao, and J. Zhang, "Recent advances in morphological cell image analysis," Computational and mathematical methods in medicine, vol. 2012, 2012.

45. H. A. Elsalamony, "Sickle anemia and distorted blood cells detection using hough transform based on neural network and decision tree," in
Proceedings of the International Conference on Image Processing, Computer Vision, and Pattern Recognition (IPCV). Computer, 2014, p. 1.

46. M. Xu, D. P. Papageorgiou, S. Z. Abidi, M. Dao, H. Zhao, and G. E. Karniadakis, "A deep convolutional neural network for classification of red blood cells in sickle cell anemia," PLoS computational biology, vol. 13, no. 10, 2017.

47. L. Alzubaidi, O. Al-Shamma, M. A. Fadhel, L. Farhan, and J. Zhang, "Classification of red blood cells in sickle cell anemia using deep convolutional neural network," in International Conference on Intelligent Systems Design and Applications. Springer, pp. 550-559, 2018.

48. T. S. Chy and M. A. Rahaman, "Automatic sickle cell anemia detection using image processing technique," 2018 International Conference on Advancement in Electrical and Electronic Engineering (ICAEEE). IEEE, pp. 1-4, 2018.

49. R. C. Gonzalez and R. E. Woods, "Digital imaging processing," Massachusetts: Addison-Wesley, 1992

\section{AUTHORS PROFILE}

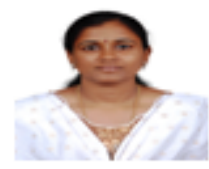

Aruna N S is a Research Scholar in Department of Electrical Engineering at College of Engineering Trivandrum, Kerala, India. She completed her M.Tech in Control Systems (2009) at College of Engineering Trivandrum and B.Tech in Electrical and Electronics Engineering (2006) at Mar Baselios College of Engineering and Technology, Kerala. She has published many works in International Journals. Her area of interest is Bio-medical engineering, Pattern Recognition and Digital Image Processing.

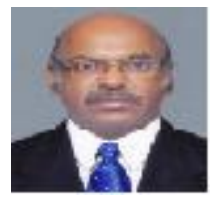

Dr. Hariharan S. is a Professor(Retd.) in the Department of Electrical Engineering at College of Engineering Trivandrum, Kerala, India. He received $\mathrm{PhD}$ from IIT, Kharagpur (2002) and M.Tech in Bio-medical Engineering from IIT, Bombay (1992) He has published many papers in Internationa Journals. Bio-medical Engineering, Pattern Recognition, Image processing, Electrical Machines are his areas of interest.
Published By:

Blue Eyes Intelligence Engineering
191 and Sciences Publication (BEIESP) (C) Copyright: All rights reserved.

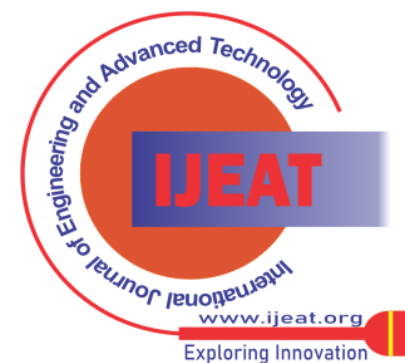

